\title{
Secondary sclerosing cholangitis in critically ill patients: current perspectives
}

\author{
Hafsteinn O Gudnason' \\ Einar S Björnsson ${ }^{1,2}$ \\ 'Division of Gastroenterology and \\ Hepatology, Department of Internal \\ Medicine, Landspitali, University \\ Hospital of Iceland, ${ }^{2}$ Faculty of \\ Medicine, University of Iceland, \\ Reykjavik, Iceland
}

This article was published in the following Dove Press journal:

Clinical and Experimental Gastroenterology

23 June 2017

Number of times this article has been viewed

\begin{abstract}
Secondary sclerosing cholangitis (SSC) is a term used for a group of chronic cholestatic disease affecting the intra- and/or extrahepatic biliary tree with inflammation and progressive stricture formation, which can lead to biliary cirrhosis. A newly recognized form of SSC is secondary sclerosing cholangitis in critically ill patients (SSC-CIP). Pathogenesis is believed to involve ischemic injury of intrahepatic bile ducts associated with prolonged hypotension, vasopressors administration, and/or mechanical ventilation in patients treated in the intensive care unit (ICU). Patients diagnosed with SSC-CIP have no prior history of liver disease and no known pathologic process or injury responsible for bile duct obstruction prior to ICU treatment. Reasons leading to ICU treatment are many including multitrauma, burn injury, cardiac surgery, severe pneumonia, other infections, or bleeding after abdominal surgery. Patients have in common prolonged ICU admission. SSC-CIP is associated with rapid progression to liver cirrhosis and poor survival with limited treatment options except a liver transplantation. Transplant-free survival is around 17-40 months, which is lower than in other SSC patients. During the initial stages of the disease, the clinical symptoms and biochemical profile are not specific and easily missed. Biliary casts formation may be considered pathognomonic for SSC-CIP since most patients have them in early stages of the disease. Increased awareness and early detection of the disease and its complications is considered to be crucial to improve the poor prognosis.
\end{abstract}

Keywords: secondary sclerosing cholangitis, SSC-CIP, chronic cholestatic disease, sclerosing cholangitis

\section{Introduction}

Sclerosing cholangitis is a group of chronic cholestatic disorders affecting the intraand/or extrahepatic biliary tree, which can lead to biliary cirrhosis and liver failure. ${ }^{1-11}$ Sclerosing cholangitis can be divided into the more well-known primary sclerosing cholangitis (PSC), which is an idiopathic form mostly associated with inflammatory bowel disease, ${ }^{12,13}$ and secondary sclerosing cholangitis (SSC), the acquired form where the etiology can usually be identified. ${ }^{14}$ Infections, toxicity, and ischemia of the bile ducts are the most common causes of SSC. ${ }^{1-11}$ A recently recognized form of SSC has been observed in patients after long treatment in intensive care units (ICUs) called secondary sclerosing cholangitis in critically ill patients (SSC-CIP). As critical care medicine keeps improving, it results in more patients treated and surviving intensive care. This results in increasing reports of ICU-associated diseases, including SSC-CIP. ${ }^{14,15}$

In severe form of SSC-CIP, treatment options are limited to liver transplantations (LT), although this is a rare indication for LT. SSC-CIP is a relatively newly described condition with one of the first cases reported in the year 1997 by Schmitt et al ${ }^{16}$ and 
others have confirmed theses observations. ${ }^{17,18}$ SSC-CIP is most likely under diagnosed and under reported. ${ }^{17,18}$ This review is meant to be a state-of-the-art on the pathogenesis, diagnosis, treatment, and outcomes of patients with SSC-CIP.

\section{Patient characteristics}

The prerequisite for the diagnosis of SSC-CIP is a lack of prior history of liver disease and no known pathologic process or injury responsible for bile duct obstruction. ${ }^{16-21}$ Patients have had in common a prolonged need for ICU treatment for a mean of 30-40 days ${ }^{14,15,18}$ and need for mechanical ventilation. Mean age of these patients at diagnosis ranges from 46 to 56 years old (range: 16-79 years) (Table 1). ${ }^{14,15,17,18,22,23}$ Males seem to be more susceptible to this condition as male to female ratio is around 1.3:1 to $9: 1 .^{14,15,17,18,23}$ Patients were admitted for prolonged ICU treatment for a variety of conditions, for example, polytrauma, burn injury, cardiac surgery, subarachnoid hemorrhage, severe pneumonia, acute respiratory distress syndrome (ARDS), infections, or bleeding after abdominal surgery. ${ }^{14,17,18,22-24}$

\section{Pathogenesis}

Two major concepts are thought to be the underlying pathophysiological mechanism of SSC-CIP. The concept receiving the most attention is the so called "concept of ischemic cholangiopathy". The hepatocytes of the liver receive dual blood supply, both from the portal vein and from the hepatic arteries. On the other hand, the biliary epithelium receives blood supply solely from the peribiliary plexus, which is composed of branches from the hepatic arteries. As a consequence, the biliary epithelium is much more susceptible to ischemia than the hepatocytes. ${ }^{15,17}$ Our knowledge on the role of ischemia of the bile ducts is mostly based on liver transplant patients. ${ }^{25}$ Early after LT, hepatic artery thrombosis, a well-known complication after LT can lead to interruption of the arterial blood supply resulting in bile duct necrosis. ${ }^{15,25}$ Ludwig et $\mathrm{al}^{7}$ also showed that administration of the chemotherapy drug, floxuridine, in the hepatic artery resulted in bile duct necrosis and sclerosing cholangitis. Ischemia of the biliary epithelium can occur when the blood supply to the bile ducts is reduced resulting in bile cast formation due to necrosis and SSC as Ludwig et al reported. ${ }^{7,15,17}$ Chemical analysis has revealed proteins as the main composition of these biliary casts supporting that it is due to necrosis of the biliary epithelium. ${ }^{17}$

The other concept is the so called concept of toxic bile. This concept focuses on the destruction of protective mechanisms for cholangiocytes. ${ }^{15}$ Without protection, the detergent properties of hydrophobic bile acids might destroy the lipid membrane of cholangiocytes. The main protective mechanism is secretion of phospholipids from the hepatocytes via the transporter lipid export pump MDR3, which form protective mixed micelles with bile acids. Also the biliary secretion of bicarbonate, via the transporter anion exchanger 2 (AE2), forms a protective alkaline bicarbonate film on the apical cholangiocyte membrane as a part of defense strategy. ${ }^{26} \mathrm{~A}$ disturbance in the fine balance between bile acids and protective mechanisms can lead to damage to the biliary epithelium, which in turn can lead to sclerosing cholangitis. This balance is believed to be threatened in critically ill patients by various conditions such as systemic inflammatory response syndrome (SIRS)/sepsis and severe hypotension. ${ }^{15,27}$ It has been proposed that individuals with genetic variants of the transporter lipid export pump MDR3 may have predisposition to formation of toxic bile under conditions of ischemia

Table I Summary of published case series of SSC-CIP with number of patients, male to female ratio, age at diagnosis, liver transplantations performed, and mortality

\begin{tabular}{|c|c|c|c|c|c|}
\hline Authors, year & Patients, $\mathbf{n}$ & $\begin{array}{l}\text { Male:female } \\
\text { ratio }\end{array}$ & $\begin{array}{l}\text { Mean age at diagnosis } \\
\text { (years) }\end{array}$ & LT, n (\%) & $\begin{array}{l}\text { Mortality, } \\
\text { n (\%) }\end{array}$ \\
\hline Scheppach et al, 200I ${ }^{43}$ & 3 & $\mathrm{I}: 2$ & $32 \pm 7$ & $\mathrm{I}(33)$ & $0(0)$ \\
\hline Engler et al, $2003^{23}$ & 9 & $5: 4$ & $56 \pm 17$ & $I(I I)$ & $5(56)$ \\
\hline Benninger et al, $2005^{6}$ & 5 & $4: 1$ & $48 \pm 20$ & $0(0)$ & $I(20)$ \\
\hline Jaeger et al, $2006^{19}$ & 10 & $5: 5$ & $55(M)$ & $I(I 0)$ & $I(10)$ \\
\hline Gelbmann et al, $2007^{17}$ & 26 & $20: 6$ & $47 \pm 18$ & $4(15)$ & $7(27)$ \\
\hline Esposito et al, $2008^{14}$ & 10 & $9: 1$ & $50 \pm 17$ & $I(10)$ & $6(60)$ \\
\hline Kulasiz et al, $2008^{42}$ & 29 & $21: 8$ & $52(M)$ & $3(10)$ & $19(66)$ \\
\hline Kirchner et al, $201 \mathrm{I}^{22}$ & 11 & $10: 1$ & $46 \pm 12$ & $11(100)$ & $4(36)$ \\
\hline Voightlander et al, $2012^{20}$ & 54 & $\mathrm{n} / \mathrm{a}$ & $50(M)$ & $6(I I)$ & $27(50)$ \\
\hline Weig et al, $2012^{44}$ & 5 & $5: 0$ & $46 \pm 10$ & $0(0)$ & $5(100)$ \\
\hline Ben-Ari et al, $2015^{24}$ & 4 & $4: 0$ & $38 \pm 18$ & I (25) & $2(50)$ \\
\hline Leonhardt et al, $2015^{18}$ & 16 & $11: 5$ & $46 \pm 15$ & $9(56)$ & $3(19)$ \\
\hline
\end{tabular}

Note: Mean age is presented with standard deviation.

Abbreviations: SSC-CIP, sclerosing cholangitis in critically ill patients; LT, liver transplantation; M, median; n/a, not available. 
or inflammatory stress. ${ }^{28}$ This might explain why some ICU patients develop SSC-CIP and others with similar disease states recover without biliary deterioration.

\section{Trigger mechanisms}

A number of trigger factors have been proposed as causes for the development of SSC-CIP, including prolonged hypotension, vasopressors administration, and techniques in mechanical ventilation. ${ }^{15}$ Leonhardt et a ${ }^{15}$ noted that all their patients experienced an episode of severe hemodynamic instability with a decrease in mean arterial blood pressure (MAP) below $65 \mathrm{mmHg}$ lasting for at least 60 minutes and often longer. They also noted that the onset of cholestasis was most closely time related in the time interval between severe hypotension and cholestasis. ${ }^{15}$ Ben-Ari et a ${ }^{24}$ also observed severe hypotension (less than $70 \mathrm{mmHg}$ in systolic pressure) in all their patients.

Vasopressors administration is a common treatment in the ICU patients before the diagnosis of SSC-CIP. ${ }^{15,29}$ Epinephrine, norepinephrine, dopamine, and dobutamine all increase systemic blood pressure but do not have the same effect on hepatosplanchnic blood flow. Dopamine has been shown to have positive effect on liver perfusion. ${ }^{30}$ In contrast, epinephrine and norepinephrine are believed to have a negative effect on splanchnic blood flow. ${ }^{31}$ Although catecholamines are believed to play a role in the pathogenesis of SSC-CIP, high-dose catecholamine ( $>0.2 \mu \mathrm{g} / \mathrm{kg} / \mathrm{min})$ do not seem to cause it, and although theoretically possible, there is a lack of experimental proof for this effect. ${ }^{15}$

Duration of mechanical ventilation in these patients has been reported from approximately 26 to 41 days. ${ }^{15,17,23}$ The $\mathrm{PaO}_{2} / \mathrm{FiO}_{2}$ ratio was $<150 \mathrm{mmHg}$ in all patients in one study and in $7 / 16$ patient in another. ${ }^{15}$ All needed $>10 \mathrm{cmH}_{2} \mathrm{O}$ positive end-expiratory pressures (PEEP) in one study ${ }^{17}$ and $50 \%$ of patients in another. ${ }^{15} \mathrm{~A}$ meta-analysis by Lin et $\mathrm{al}^{29}$ showed that all patients had high PEEP treatment with average treatment duration of 36 days and mean pressure of PEEP $12.8 \mathrm{~cm} \mathrm{H}_{2} \mathrm{O}$. Gelbmann et $\mathrm{al}^{17}$ also noted that many patients needed high-frequency oscillatory ventilation ( $9 / 17$ patients) and intermittent prone positioning (12/17 patients). It has been proposed that lung protective mechanical ventilation with high PEEP, low tidal volume, and prone positioning can decrease splanchnic blood flow. ${ }^{32,33}$

\section{Microbiological examination}

In about $98 \%$ of SSC-CIP patients, bacteria and/or Candida species are detectable in the collected bile. ${ }^{21}$ Gelbmann et a ${ }^{17}$ performed a study on bile cultures collected through endoscopic retrograde cholangiopancreatography (ERCP) from patients with SSC-CIP. The test revealed growth of Enterococcus faeceum or Enterococcus faecalis in $71 \%$ of patients. A total of $67 \%$ of the isolates showed resistance to ciprofloxacin and gentamicin. ${ }^{17}$ In addition, half of the isolates showed resistance to imipenem and amoxicillinclavulanic acid. Other isolates were candida, methicillinresistant Staphylococcus aureus, Pseudomonas aeruginosa, and Stenotrophomonas maltophilia. ${ }^{17}$ Other studies have confirmed that Enterococcus and candida albicans are the main isolated microorganism in the bile. ${ }^{22}$ Cultures from drained abscesses in patients with SSC-CIP after burn injuries revealed hospital-acquired multiple-resistant bacteria such as P. aeruginosa and Acinobacter. ${ }^{24}$ It is unclear whether these pathogens play a role in the pathogenesis or just incidental findings of innocent bystanders or colonizers. At least they show a high rate of antibiotic resistance, which must be taken into count during the treatment of SSC-CIP patients.

\section{Diagnosis}

\section{Clinical symptoms}

In the initial stages of SSC, clinical symptoms are rare, and the only indication of cholestasis is elevated liver tests. This often leads to a delayed or a missed diagnosis. Patients with persistent cholestasis should be suspected having sclerosing cholangitis. The differential diagnoses among patients in the ICU with cholestatic liver tests are several such as cholestasis associated with sepsis, ${ }^{34}$ cholangitis due to choledocholithiasis, ${ }^{35}$ and drug-induced liver injury (DILI) ${ }^{36}$ The most common type of agents leading to idiosyncratic liver injury are antibiotics, ${ }^{36,37}$ and these are commonly used in the ICU. Thus, DILI is an important differential diagnosis in these patients, particularly in those with normal hepatobiliary imaging. As disease progresses, clinical symptoms develop. The most common symptoms are jaundice, pruritus, and abdominal discomfort localized in the right upper quadrant of the abdomen. ${ }^{38,39}$ Recurrent biliary infections are typically observed in SSC patients. ${ }^{39}$ Patients with SSC-CIP present with persisting symptoms of cholestasis, despite recovery from their primary illness that needed ICU treatment. This is the main clinical finding that differentiates SSC-CIP patients from patients with endotoxin-associated jaundice, which resolves after clinical recovery. ${ }^{39}$

\section{Liver tests and other laboratory parameters}

No specific clinical and biochemical parameter indicates the presence of early sclerosing cholangitis. Major presenting 
signs are persistent elevated gamma-glutamyl transferase (GGT) (peak 20-50 times upper limit of normal [ULN]) and alkaline phosphatase (ALP) (peak 5-21 times ULN) ${ }^{18}$ along with elevated bilirubin levels (peak 3-39 times ULN) (Table 2). ${ }^{17}$ GGT is the first parameter to become elevated, usually around 7 days after initial life-threatening event, followed by ALP elevation within a few days. Bilirubin is the last of the three to become elevated showing a mean of 16 days after initial life-threatening event. ${ }^{18}$ While these three parameters can become severely elevated, alanine aminotransferase (ALT) and aspartate aminotransferase (AST) are usually only mildly or moderately elevated. ${ }^{18}$ Another finding made by Leonhardt et $\mathrm{al}^{18}$ was that cholesterol levels was significantly elevated, with a mean of $536 \mathrm{mg} / \mathrm{dL}$, compared to a normal value of $200 \mathrm{mg} / \mathrm{dL}$ within the first year of disease.

\section{Imaging}

ERCP has been considered to be the gold standard for diagnosing SSC-CIP. Compared with ERCP, the accuracy for diagnosing SSC-CIP was 30\% for ultrasound and 36\% for liver biopsies. ${ }^{20}$ In recent years, magnetic resonance cholangiopancreatography (MRCP) has been increasingly utilized as a diagnostic instrument and is preferred, prior to an ERCP, since it is noninvasive imaging and these are critically ill patient at risk when undergoing any invasive procedure. ${ }^{24}$ Common initial findings during the early stage of the disease on ERCP are multiple ribbon-like intraductal filling defects known as biliary cast formation. ${ }^{18,40}$ These are followed by infections that can lead to multiple hepatic abscesses. In later stages, findings are multiple irregular strictures, dilations, beading, wall thickening, and destruction of the intrahepatic bile ducts with sparing of the common bile duct forming a "pruned tree" appearance. ${ }^{18,24,41}$ All patients have intrahepatic bile duct involvement, whereas only around a fifth of patients also have minor extrahepatic disease. ${ }^{18}$ Leonhardt et al ${ }^{18}$ found out that a total of $88 \%$ of SSC-CIP patient developed imaging features of liver cirrhosis within 6 months of diagnosis. There is often a delay until an ERCP is performed, and final diagnosis of SSC-CIP has usually not been made until 2-3 months after initial signs of cholestasis. ${ }^{18,22,24}$

\section{Histopathology}

Esposito et al ${ }^{14}$ examined 10 liver biopsies of patients diagnosed with SSC-CIP taken 1.5-57 months after diagnosis. Morphological changes in the portal area include inflammatory-degenerative changes in the bile ducts, mild to moderate periductal inflammatory infiltrate, portal edema with moderate portal enlargement, cholestatic features with ductular reaction, and cholate stasis and fibrosis without a specific periductal pattern. ${ }^{14}$ Acinar changes include hepatocellular and/or canalicular cholestasis, perivenular necroses, and bile infarcts. ${ }^{14}$ These results are comparable to the results of Gelbmann et $\mathrm{al}^{17}$ and Leonhardt et al. ${ }^{18}$ Gelbmann et al noted that during the early phase, biopsy findings were suggestive of bile duct "obstruction", including periportal bile duct infarcts and perivenular bilirubinostasis with bile pluggins of dilated canaliculi. Ductular proliferation, portal fibrosis, and thrombotic occlusion of the portal hepatic arteries or veins were absent. Within 4-12 months after beginning of cholestasis, biopsies showed severe histological alterations including bile duct proliferation, portal inflammatory infiltrate, portal, and periductular fibrosis. Finally after 14-17 months, one of two biopsies showed incomplete liver cirrhosis. It was concluded that progression to liver fibrosis seems to be dependent on the extent of initial biliary injury. ${ }^{17,18}$ A persistent infection of the bile ducts also often aggravates the fibrotic process. ${ }^{17}$ Studies have not shown any correlation between laboratory values and morphological evidence of cholestasis. ${ }^{14}$

\section{Complications of SSC-CIP}

The outcome of patients with SSC-CIP is often liver cirrhosis and hepatic failure with high mortality rate. ${ }^{20,42}$ However, other complications can arise during the disease process that must be taken into consideration as some of these are treatable. Acalculous cholecystitis has been noted in over half of patients with SSC-CIP within the first year and around half of those require emergency cholecystectomy. ${ }^{18}$ It seems that

Table 2 Summary of published case series with peak liver parameters of patients with SSC-CIP

\begin{tabular}{llllll}
\hline Authors, year & ALP (U/L) & GGT (U/L) & Bilirubin (mg/dL) & ALT & AST \\
\hline Engler et al, 200323 & 1660 & 445 & 10.2 & $\mathrm{n} / \mathrm{a}$ & $\mathrm{n} / \mathrm{a}$ \\
Gelbmann et al, 2007'7 & $1182 \pm 1058$ & $\mathrm{n} / \mathrm{a}$ & $10.4 \pm 9.1$ & $\mathrm{n} / \mathrm{a}$ & $\mathrm{n} / \mathrm{a}$ \\
Kulasiz et al, 200842 & $758 \pm 544$ & $726 \pm 815$ & $12.3 \pm 10.6$ & $172 \pm 191$ & $171 \pm 184$ \\
Ben-Ari et al, 201524 & $2484 \pm 496$ & $4045 \pm 2057$ & $19.7 \pm 15.2$ & $158 \pm 29$ & $207 \pm 42$ \\
\hline
\end{tabular}

Note: All liver parameters are presented as mean with standard deviation if available.

Abbreviations: SSC-CIP, sclerosing cholangitis in critically ill patients; ALP, alkaline phosphatase; GGT, gamma-glutamyl transferase; ALT, alanine aminotransferase; AST, aspartate aminotransferase; n/a, not available. 
the gallbladder epithelium is involved in the pathogenesis process of SSC-CIP. Therefore, SSC-CIP should be suspected in ICU patients with acalculous cholecystitis and unexplained cholestasis. ${ }^{18,23}$

Bacterial cholangitis is common in patients with SSCCIP, which is probably due to strictures and destruction of segmental and subsegmental bile ducts. As a result of ductal damage, peripheral bile duct branches lose their connection to the central ducts. The elimination of a biliary obstruction is needed for successful antibiotic treatment of cholangitis, and excluded ducts are hard to drain and therefore cannot receive penetration from antibiotics. This limits the effectiveness of antibiotic treatment and increases the risk of so-called cholangiosepsis with high mortality rate. Furthermore, the stagnation of bile flow promotes cholangitic liver abscesses. ${ }^{18}$

Leonhardt et $\mathrm{al}^{18}$ noted that patients had severe weight loss of mean $18 \mathrm{~kg}$ within first year after initial injury, which was though not associated with the length of stay in the ICU.

\section{Treatment}

Treatment options for patients with SSC-CIP are unfortunately rather limited. Patients with clinical evidence of bacterial cholangitis are treated with broad spectrum antibiotics according to microbiologic testing if available. ${ }^{42}$ Various different endotherapies, including endoscopic dilations, endoscopic sphincterotomy of the sphincter of Oddi, intermittent stenting, removal of biliary casts in the larger intrahepatic bile ducts with a dormia basket, and sludge extraction, have been performed in patients diagnosed with SSC-CIP. ${ }^{19,23,29,42}$ Patients have biliary cast formations that are black-pigmented and necrotic material that obstruct the bile flow and increase risk of infection. The cast formations can in some cases be extracted during ERCP procedure. ${ }^{19,42}$ In patients with casts in the smaller bile ducts that cannot be reached during normal ERCP procedure a continuous saline rinsing through a nasobiliary drainage for several days has been performed. However, saline rinsing has not shown much success in removing more of the intraductal material due to the insolubility and the adherence of the casts. ${ }^{17}$ These different procedures have shown to be associated with lower bilirubin and ALP levels and short-term clinical improvement, although controlled studies are lacking. However, these measures do not seem to prevent the progressive destruction of the biliary tree and progression of the disease. ${ }^{17,23}$ Ursodeoxycholic acid (UDCA) administration has also been given, although it has not been proven to reduce mortality. ${ }^{23,29,40,42} \mathrm{Up}$ to $75 \%$ of patients have to be placed on the waiting list for liver transplant within the first year after the onset of cholestasis. ${ }^{18}$

\section{Outcome and prognosis}

SSC-CIP is a progressive cholestatic liver condition characterized by necrosis of the bile duct epithelium, which leads to rapid destruction of intrahepatic bile ducts. When manifested, it usually runs a chronic, irreversible course, and the ultimate cure in the most severe cases is only LT. Transplant-free survival is around $17-40$ months. ${ }^{18,42}$ One-year survival rate after LT for patients with SSC-CIP is approximately $85 \%$, which is comparable with that of patients transplanted due to alcoholic liver cirrhosis. ${ }^{18,22}$ Without transplantation, 1-year survival is as poor as $55 \%$ and only $14 \%$ after 6 years. ${ }^{42}$ Compared to PSC and SSC in general, the survival is significantly shorter for SSC-CIP, with a median survival of 13 months for SSCCIP versus 72 months for SSC in general and 89 months for PSC patients. ${ }^{40}$ The most common cause of mortality is hepatic failure, and around half of patient who survive have cholestatic cirrhosis in a stable condition, and the rest are waiting for $\mathrm{LT}^{29}$

\section{Conclusion}

$\mathrm{SSC}-\mathrm{CIP}$ is a rather newly recognized condition that has been gaining more attention in recent years due to rise in ICU treatment-related diseases with improved ICU treatment. SSC-CIP is usually a progressive disease with an aggressive nature leading to liver cirrhosis and hepatic failure without LT. $^{42}$ During the initial stages of the disease, the clinical symptoms and biochemical profile are not specific and do not differentiate much from endotoxin-associated jaundice, which is a common condition in critically ill patients. Thus, increased awareness and early detection of SSC-CIP and its complications are believed to be crucial to improve the poor prognosis. Biliary casts formation may be considered pathognomonic for SSC-CIP since most patients have them in early stages of the disease and these casts have not been described in the literature in either PSC or IgG4 cholangiopathy. ${ }^{18}$

\section{Disclosure}

The authors report no conflicts of interest in this work.

\section{References}

1. Imam MH, Talwalkar JA, Lindor KD. Secondary sclerosing cholangitis: pathogenesis, diagnosis, and management. Clin Liver Dis. 2013;17(2):269-277.

2. Forbes A, Blanshard C, Gazzard B. Natural history of AIDS related sclerosing cholangitis: a study of 20 cases. Gut. 1993;34(1):116-121.

3. Watanabe $\mathrm{H}$, Ohira $\mathrm{H}$, Kuroda M, et al. Primary sclerosing cholangitis with marked eosinophilic infiltration in the liver. J Gastroenterol. 1995;30(4) 524-528.

4. Sperling RM, Koch J, Sandhu JS, Cello JP. Recurrent pyogenic cholangitis in Asian immigrants to the United States: natural history and role of therapeutic ERCP. Dig Dis Sci. 1997;42(4):865-871. 
5. Bjornsson E, Chari ST, Smyrk TC, Lindor K. Immunoglobulin G4 associated cholangitis: description of an emerging clinical entity based on review of the literature. Hepatology. 2007;45(6):1547-1554.

6. Benninger J, Grobholz R, Oeztuerk Y, et al. Sclerosing cholangitis following severe trauma: description of a remarkable disease entity with emphasis on possible pathophysiologic mechanisms. World $J$ Gastroenterol. 2005;11(27):4199-4205.

7. Ludwig J, Kim CH, Wiesner RH, Krom RA. Floxuridine-induced sclerosing cholangitis: an ischemic cholangiopathy? Hepatology. 1989;9(2):215-218.

8. Schwab GP, Wetscher GJ, Vogl W, Redmond E. Methimazole-induced cholestatic liver injury, mimicking sclerosing cholangitis. Langenbecks Arch Chir. 1996;381(4):225-227.

9. Wong SW, Lee KF, Wong J, Ng WW, Cheung YS, Lai PB. Dilated common bile ducts mimicking choledochal cysts in ketamine abusers. Hong Kong Med J. 2009;15(1):53-56.

10. Seto WK, Ng M, Chan P, et al. Ketamine-induced cholangiopathy: a case report. Am J Gastroenterol. 2011;106(5):1004-1005.

11. Gudnason HO, Bjornsson HK, Gardarsdottir M, et al. Secondary sclerosing cholangitis in patients with drug-induced liver injury. Dig Liver Dis. 2015;47(6):502-507.

12. Broome U, Olsson R, Loof L, et al. Natural history and prognostic factors in 305 Swedish patients with primary sclerosing cholangitis. Gut. 1996;38(4):610-615.

13. de Valle MB, Bjornsson E, Lindkvist B. Mortality and cancer risk related to primary sclerosing cholangitis in a Swedish population-based cohort. Liver Int. 2012;32(3):441-448.

14. Esposito I, Kubisova A, Stiehl A, Kulaksiz H, Schirmacher P. Secondary sclerosing cholangitis after intensive care unit treatment: clues to the histopathological differential diagnosis. Virchows Arch. 2008;453(4):339-345.

15. Leonhardt S, Veltzke-Schlieker W, Adler A, et al. Trigger mechanisms of secondary sclerosing cholangitis in critically ill patients. Crit Care. 2015;19:131.

16. Schmitt M, Kolbel CB, Muller MK, Verbeke CS, Singer MV. [Sclerosing cholangitis after burn injury]. Z Gastroenterol. 1997;35(10): 929-934.

17. Gelbmann CM, Rummele P, Wimmer M, et al. Ischemic-like cholangiopathy with secondary sclerosing cholangitis in critically ill patients. Am J Gastroenterol. 2007;102(6):1221-1229.

18. Leonhardt S, Veltzke-Schlieker W, Adler A, et al. Secondary sclerosing cholangitis in critically Ill patients: clinical presentation, cholangiographic features, natural history, and outcome: a series of 16 cases. Medicine. 2015;94(49):e2188.

19. Jaeger C, Mayer G, Henrich R, et al. Secondary sclerosing cholangitis after long-term treatment in an intensive care unit: clinical presentation, endoscopic findings, treatment, and follow-up. Endoscopy. 2006;38(7):730-734.

20. Voigtlander T, Negm AA, Schneider AS, et al. Secondary sclerosing cholangitis in critically ill patients: model of end-stage liver disease score and renal function predict outcome. Endoscopy. 2012;44(11):1055-1058.

21. Kirchner GI, Rummele P. Update on sclerosing cholangitis in critically Ill patients. Viszeralmedizin. 2015;31(3):178-184.

22. Kirchner GI, Scherer MN, Obed A, et al. Outcome of patients with ischemic-like cholangiopathy with secondary sclerosing cholangitis after liver transplantation. Scand J Gastroenterol. 2011;46(4):471-478.

23. Engler S, Elsing C, Flechtenmacher C, Theilmann L, Stremmel W, Stiehl A. Progressive sclerosing cholangitis after septic shock: a new variant of vanishing bile duct disorders. Gut. 2003;52(5):688-693.
24. Ben-Ari Z, Levingston D, Weitzman E, et al. Secondary sclerosing cholangitis following major burn. Ann Hepatol. 2015;14(5):695-701.

25. Mourad MM, Liossis C, Gunson BK, et al. Etiology and management of hepatic artery thrombosis after adult liver transplantation. Liver Transpl. 2014;20(6):713-723.

26. Beuers U, Hohenester S, de Buy Wenniger LJ, Kremer AE, Jansen PL, Elferink RP. The biliary $\mathrm{HCO}(3)(-)$ umbrella: a unifying hypothesis on pathogenetic and therapeutic aspects of fibrosing cholangiopathies. Hepatology. 2010;52(4):1489-1496.

27. Popov Y, Patsenker E, Fickert P, Trauner M, Schuppan D. Mdr2 (Abcb4)-/- mice spontaneously develop severe biliary fibrosis via massive dysregulation of pro- and antifibrogenic genes. $J$ Hepatol. 2005;43(6):1045-1054.

28. Trauner M, Fickert P, Wagner M. MDR3 (ABCB4) defects: a paradigm for the genetics of adult cholestatic syndromes. Semin Liver Dis. 2007;27(1):77-98.

29. Lin T, Qu K, Xu X, et al. Sclerosing cholangitis in critically ill patients: an important and easily ignored problem based on a German experience. Front Med. 2014;8(1):118-126.

30. Hiltebrand LB, Krejci V, Sigurdsson GH. Effects of dopamine, dobutamine, and dopexamine on microcirculatory blood flow in the gastrointestinal tract during sepsis and anesthesia. Anesthesiology. 2004;100(5): 1188-1197.

31. Meier-Hellmann A, Reinhart K, Bredle DL, Specht M, Spies CD, Hannemann L. Epinephrine impairs splanchnic perfusion in septic shock. Crit Care Med. 1997;25(3):399-404.

32. Putensen $\mathrm{C}$, Wrigge $\mathrm{H}$, Hering $\mathrm{R}$. The effects of mechanical ventilation on the gut and abdomen. Curr Opin Crit Care. 2006;12(2):160-165.

33. Fujita Y. Effects of PEEP on splanchnic hemodynamics and blood volume. Acta Anaesthesiol Scand. 1993;37(4):427-431.

34. Fuchs M, Sanyal AJ. Sepsis and cholestasis. Clin Liver Dis. 2008;12(1):151-172,ix.

35. Hanau LH, Steigbigel NH. Acute (ascending) cholangitis. Infect Dis Clin North Am. 2000;14(3):521-546.

36. Bjornsson ES, Bergmann OM, Bjornsson HK, Kvaran RB, Olafsson S. Incidence, presentation, and outcomes in patients with drug-induced liver injury in the general population of Iceland. Gastroenterology. 2013;144(7):1419-25, 1425.e1-3. quiz e1419-1420.

37. Bjornsson ES. Drug-induced liver injury due to antibiotics. Scand J Gastroenterol. 2017;20:1-7.

38. Deltenre P, Valla DC. Ischemic cholangiopathy. J Hepatol. 2006;44(4): 806-817.

39. Ruemmele P, Hofstaedter F, Gelbmann CM. Secondary sclerosing cholangitis. Nat Rev Gastroenterol Hepatol. 2009;6(5):287-295.

40. Gossard AA, Angulo P, Lindor KD. Secondary sclerosing cholangitis: a comparison to primary sclerosing cholangitis. Am J Gastroenterol. 2005;100(6):1330-1333.

41. Weiss KE, Willmann JK, Jeffrey RB, Desser TS. Secondary sclerosing cholangitis in a critically ill patient. Quant Imaging Med Surg. 2016;6(2): 224-228.

42. Kulaksiz H, Heuberger D, Engler S, Stiehl A. Poor outcome in progressive sclerosing cholangitis after septic shock. Endoscopy. 2008;40(3):214-218.

43. Scheppach W, Druge G, Wittenberg G, et al. Sclerosing cholangitis and liver cirrhosis after extrabiliary infections: report on three cases. Crit Care Med. 2001;29(2):438-441.

44. Weig T, Schubert MI, Gruener N, et al. Abdominal obesity and prolonged prone positioning increase risk of developing sclerosing cholangitis in critically ill patients with influenza A-associated ARDS. Eur J Med Res. 2012;17:30. 
Clinical and Experimental Gastroenterology is an international, peerreviewed, open access, online journal publishing original research, reports, editorials, reviews and commentaries on all aspects of gastroenterology in the clinic and laboratory. This journal is included on PubMed. The manuscript management system is completely online and includes a very quick and fair peer-review system, which is all easy to use. Visit http://www.dovepress.com/testimonials.php to read real quotes from published authors.

Submit your manuscript here: https://www.dovepress.com/clinical-and-experimental-gastroenterology-journal 